ORIGINAL ARTICLE

\title{
Parental perceptions regarding mandatory mouthguard use in competitive youth soccer
}

\author{
J M Pribble, R F Maio, G L Freed
}

See end of article for authors' affiliations

......................

Correspondence to: Dr J M Pribble, Department of Emergency Medicine, University of Michigan, 300 NIB, 2D06, Box 0437, Ann Arbor, M 48109-0437, USA; ipribb@umich.edu
Injury Prevention 2004;10:159-162. doi: 10.1136/ip.2003.004754

Objective: To understand factors that influence parental perceptions regarding mandatory mouthguard use in competitive youth soccer.

Setting and subjects: A web based survey of parents whose 8-14 year old children participated in outdoor competitive youth soccer during the fall of 2002. Questions focused on: previous injury, education provided, dentist/physician recommendations, and parental perceptions of mouthguard use.

Main outcome measure: The outcome variable was parental agreement with the statement "mouthguards should be mandatory for competitive youth soccer". Logistic regression and $\chi^{2}$ were performed using STATA 8.0.

Results: Altogether 120 parents participated. The children had a mean (SD) age of $11.8(1.5)$ years and $48 \%$ were female; $14 \%$ wore mouthguards and $11 \%$ suffered orofacial injuries. Nineteen percent of parents reported receiving educational material from the youth soccer organization. Although $92 \%$ of parents believed that mouthguards were effective in reducing orofacial injuries, only one half agreed that mouthguards should be mandatory. Thirty percent of respondents reported that their dentist/physician recommended mouthguards for competitive youth soccer. Logistic regression showed that dentist/ physician recommendation and parental female gender were independently associated with parental agreement for mandatory mouthguard use (odds ratio $2.9,95 \%$ confidence interval (CI) 1.2 to 7.3 ; odds ratio $3.0,95 \% \mathrm{Cl} 1.1$ to 6.5 ).

Conclusions: Few athletes wear mouthguards during competitive youth soccer. Health care professionals in this study did not capitalize on the apparent influence they have on parental beliefs regarding mouthguard use. Efforts are needed by both health professionals and soccer organizations to educate parents about orofacial injuries and mouthguard use in competitive youth soccer.
S occer is the most popular sport worldwide with at least 40 million participants. ${ }^{1}$ Although soccer is not traditionally viewed as a "contact" sport, the potential for contact with other players, the ground, the ball, and the goalposts during soccer participation is significant. The National Electronic Injury Surveillance System estimated 175000 soccer related injuries were evaluated in emergency departments in 2001, with nearly $43 \%$ of these injuries suffered by participants between the ages of 8 and 14 years. ${ }^{2}$

Up to $30 \%$ of all injuries in soccer are to the orofacial region and include tooth avulsions, tooth fractures, concussions, and oral lacerations. ${ }^{3-6}$ Research has shown that mouthguard use significantly reduces orofacial injuries while playing various sports. ${ }^{7-9}$ This has led the American Dental Association (ADA) and the American Academy of Pediatric Dentistry (AAPD) to recommend mouthguard use for soccer participants. $^{10}{ }^{11}$ Despite this information, very few soccer participants wear mouthguards. ${ }^{3}{ }^{12}$

Historically, orofacial injury studies have been conducted in the adolescent and young adult population. Little is known about competitive youth soccer mouthguard use and orofacial injury. Competitive leagues commonly hold "try-outs" to fill their rosters and may increase the number of participants who are more aggressive, faster, and stronger. Moreover, little is known about differences in behavior or refereeing patterns in these age groups of soccer participants. It is unclear whether these factors increase the risk of orofacial injury to the competitive youth soccer athlete compared with their age matched counterparts participating in recreational league soccer or affect parental perception of injury risk.

In youth soccer, parents are major stakeholders and significantly influence decisions regarding play and protective equipment use. Understanding the parental attitudes regarding orofacial injury and mouthguard use is important if we are to provide effective measures to reduce these types of injuries in soccer. Previous research has assessed parental attitudes towards mouthguards in youth sports in general. ${ }^{13}{ }^{14}$ To date, no study has evaluated the parental beliefs of mouthguard use in competitive youth soccer. This study was designed to assess parental perceptions regarding orofacial injuries, barriers to mouthguard use, and factors that may influence their beliefs regarding mandatory mouthguard use in competitive youth soccer.

\section{METHODS}

Study design and population

During the fall of 2002 we conducted a web based survey of parents of children 8-14 years of age participating in organized outdoor competitive youth soccer. The study was conducted in a Midwestern suburban community with a population of approximately 110000 . Competitive soccer was defined as a league in which players participated in a selective try-out to obtain a position on the team's roster.

\section{Study content and administration}

After obtaining approval from both the youth soccer organization management and the institutional review board of the University of Michigan, a list of parental email addresses was obtained from the local youth soccer organization. This list was used to distribute the survey via the world wide web. Parents were sent an introductory letter that included a formal consent form along with the link to the survey. For parents with multiple children participating in

Abbreviations: AAPD, American Academy of Pediatric Dentistry; ADA, American Dental Association 
competitive youth soccer, the oldest child was used as the index child for this study. Parents who did not have an email address on file with the youth soccer organization or had an invalid email address were excluded from the study.

Questions were pilot tested via a personal handwritten questionnaire. One of the authors (JP) performed the pilot testing and assessed parental understanding of the questions. After pilot testing, minor changes in wording were made and the survey was developed online through a commercial survey company (Keysurvey, Stoughton, Massachusetts). The survey consisted of 34 questions. All questions, except one, allowed the participant in the survey to point and click a fixed choice answer. There was one open ended question regarding parental perceived barriers to mouthguard use in competitive youth soccer. Parental perceptions were assessed by fixed choice response statements regarding mouthguard use using a four point Likert scale. The question regarding parental beliefs about a mandatory mouthguard rule was also assessed using a four point Likert scale and dichotomized to determine percent agreement.

The survey focused on specific issues hypothesized to influence parental perceptions regarding a mandatory mouthguard rule: previous orofacial injury to index child, parent, or sibling; history of mouthguard use by parent/ guardian; educational material provided by the soccer organization or coach; and health professional recommendations to use mouthguards while participating in soccer.

The definition of injury included lip laceration, tooth fracture, tooth subluxation, tooth avulsion, and tongue laceration that occurred to the competitive youth soccer athlete within the past 12 months while participating in organized soccer.

Survey participants were able to click on a link that directly connected them to the online survey. After completion of the survey, data were directly downloaded onto a server. STATA 8.0 (College Station, Texas) was used for analysis.

A total of four email distributions of the world wide web survey were sent during a one month period. A final emailonly version was distributed to non-respondents targeting those parents with possible suboptimal connections to the web based survey. This email survey was a shortened version of the web based survey but included all major themes.

\section{Data analysis}

Independent variables hypothesized to influence parental beliefs about a mandatory mouthguard rule were analyzed using $\chi^{2}$ statistics for association. Parental perceptions were described by dichotomizing the four point Likert scale and presenting the percentage of parents who agreed with a specific statement.

Multiple logistic regression was used to determine variables that were independently associated with parent's agreement that mouthguards should be mandatory for competitive youth soccer. Variables that were significantly associated with parental agreement regarding mandatory mouthguard use by univariate analysis along with variables hypothesized to influence this agreement were included into the regression model. A priori variables hypothesized to influence parental beliefs regarding mandatory mouthguard use were previous injury to index child, sibling, or self (parent); gender of competitive youth soccer athlete as well as parent; education about mouthguard use; and health professional recommendations to use mouthguards while participating in soccer.

\section{RESULTS}

Of 280 potential participants, 11 were excluded due to no email address on file with the youth soccer association, and
Table 1 Mean age of competitive youth soccer (CYS) athletes and percentage of parental respondents who reported affirmative to various independent variables $(n=120)$

\begin{tabular}{ll}
\hline Independent variables & \\
\hline Mean (SD) index CYS athlete age (years) & 11.8 (1.5) \\
Index CYS athlete gender (\% female) & 48 \\
Index CYS athlete injury (\%) & 11 \\
Index CYS athlete mouthguard use (\%) & 14 \\
Index CYS athlete type of mouthguard (\% custom) & 64 \\
Sibling history of orofacial injury (\%) & 12 \\
Parent gender (\% female) & 65 \\
Parent history of orofacial injury & 5 \\
Parent mouthguard use total (\%): & 14 \\
Female & 0 \\
Male & 40 \\
Parent youth sports participation total (\%): & 58 \\
Female & 32 \\
Male & 81 \\
Education regarding mouthguards provided to & 19 \\
parents (\%) & \\
Health professional recommendation to use & 30 \\
mouthguards while participating in CYS (\%) & \\
League (\% division 2) & 65 \\
\hline
\end{tabular}

${ }^{*}$ Two competitive leagues comprised the study sample. Division 2 is the more advanced of the two leagues per the youth soccer organization.

Table 2 Reported number of orofacial injuries by type in competitive youth soccer

\begin{tabular}{ll}
\hline Lip laceration & 10 \\
Mouth laceration (not lip) & 2 \\
Bleeding gums & 1 \\
Chipped tooth & 1 \\
Other* & 2 \\
\hline \multirow{2}{*}{ *Orthodontic appliance knocked loose, swollen lips. }
\end{tabular}

21 were excluded due to invalid email address. Therefore, 248 parents made up our study population.

Of these $248,48 \%(\mathrm{n}=120)$ returned completed usable surveys. Sixty five percent of the surveys were completed by the athlete's female parent/guardian. Fourteen percent of parents reported that their child wore a mouthguard during competitive youth soccer participation. Table 1 demonstrates the mean age of the index athlete along with the percentage of responding parents who answered in the affirmative to the various independent variables. Univariate analysis demonstrated that dentist/physician recommendations $(p=0.008)$ and female gender $(\mathrm{p}=0.008)$ of the responding parent were significantly associated with parental agreement about the need for a mandatory mouthguard rule in competitive youth soccer. Table 2 shows the types of orofacial injuries that were reported. Of those reporting injuries, none reported the need for acute medical/dental attention.

Fifty five percent of parents stated that their physician or dentist questioned them about their child's athletic participation but only $30 \%$ of the parents stated that their physicians or dentists recommended mouthguard use for their child while participating in soccer.

Very few parents reported receiving any information regarding mouthguards or orofacial injuries from the soccer association. Only 19\% stated that they received any information and the most common form of this information was verbally from either the coach $(52 \%)$ or parent manager (29\%). Parental reported barriers to mouthguard use were: discomfort $(42 \%)$, peer pressure (19\%), difficulty breathing $(17 \%)$, and the fact that mouthguard use was not mandatory $(15 \%)$. 
Table 3 Selected parental perception questions and percent agreement

\begin{tabular}{ll}
\hline & \% Agree \\
\hline $\begin{array}{l}\text { Parents have the majority of the responsibility for } \\
\text { safety of youth soccer athletes }\end{array}$ & 78 \\
$\begin{array}{l}\text { Mouthguards cost too much } \\
\text { Mouth and tooth injury is a significant problem in youth }\end{array}$ & 11 \\
soccer & 32 \\
$\begin{array}{l}\text { Making mouthguards mandatory would reduce the } \\
\text { number of mouth and tooth injuries }\end{array}$ & 86 \\
$\begin{array}{l}\text { Youth soccer players need to use mouthguards } \\
\text { If mouthguards were made mandatory in youth soccer, } \\
\text { the athlete would be more likely to use mouthguards }\end{array}$ & 62 \\
as an adolescent athlete & 83 \\
Mouthguards reduce mouth and tooth injury & 92 \\
Mouthguards should be mandatory for youth soccer athletes & 51
\end{tabular}

Over $90 \%$ of parents believed that mouthguards reduce mouth and tooth injury and $86 \%$ believed that mandatory use for youth soccer would reduce injuries. Moreover, 83\% agreed that making mouthguards mandatory for youth soccer would increase the likelihood that the athlete would use a mouthguard as an adolescent soccer athlete. However, only one half of the parents agreed that mouthguards should be made mandatory (table 3 ).

Table 4 demonstrates the multivariate logistic regression model. Health professional recommendations to use mouthguards and female parent were independently associated with agreement regarding mandatory mouthguard use in competitive youth soccer.

\section{DISCUSSION}

Very few health professionals recommended mouthguard use for youth soccer participants in our study. Despite the ADA and AAPD recommendations, only $30 \%$ of the parents reported their physician or dentist recommended mouthguard use for their child during soccer participation. Although lack of parental recall about health professional advice may have affected these results, the findings are consistent with Maestrello et al, who surveyed dentist and dental specialists regarding mouthguard recommendations for various sports. They reported that only $42 \%$ of dentists surveyed recommended mouthguard use for soccer participants. ${ }^{15}$ The reasons are unclear as to why so few health professionals follow this recommendation guideline. The risk of injury while participating in soccer increases with age and peaks in the adolescent and early adult ages. ${ }^{16}{ }^{17}$ Health professionals in our study may have felt that the risk of injury was too low for these youth athletes.

It is well documented the influence health professionals have on changing health behaviors. ${ }^{18}$ As such, more physicians and dentists need to make an effort to inform their patients about proper protective equipment for soccer athletes. The importance of this finding is underscored as our final model demonstrated that health professional recommendations were independently associated with parental agreement regarding mandatory mouthguards use competitive youth soccer. Emergency physicians and dentists are optimally positioned to educate youth soccer athletes and their parents. Many youth see the dentist for their regular check-ups and many present to the emergency department for minor injuries caused by sports and recreation. In fact, 2.6 million patients between the ages of 5 and 24 years present annually to the emergency department due to sports related injuries. ${ }^{19}$ Physicians and dentists are encouraged to use these settings to educate both the parents and youth about protective measures for sports and recreation activities. ${ }^{20}$
Table 4 Adjusted associations with outcome of parental belief that mouthguards should be mandatory for competitive youth soccer (CYS)

\begin{tabular}{lll}
\hline Independent variable & Odds ratio & $\begin{array}{l}95 \% \text { Confidence } \\
\text { interval }\end{array}$ \\
\hline $\begin{array}{l}\text { Health professional } \\
\text { recommendations }\end{array}$ & 2.94 & 1.18 to 7.27 \\
$\begin{array}{l}\text { Parent gender (female) } \\
\text { CYS athlete age }\end{array}$ & 2.95 & 1.12 to 6.49 \\
CYS athlete gender (male) & 1.03 & 0.77 to 1.38 \\
CYS athlete injury & 0.47 & 0.21 to 1.08 \\
Education provided to parents & 0.90 & 0.26 to 3.20 \\
\hline$\chi^{2}=16.86, p<0.01$, receiver operating characteristic $=0.71, \mathrm{n}=$ \\
116.
\end{tabular}

Another venue for education is the pre-participation physical examination. This encounter is explicitly designed to evaluate athletes for potential health problems that can be exacerbated by sports and to provide education on injury prevention. The pre-participation physical examination booklet states: "Asking about mouthguards may be unnecessary as they are now required in most collision sports". ${ }^{21}$ However, most soccer organizations do not have mandatory mouthguard rules. To ensure the safest possible playing environment for our youth athletes, health organizations need to provide consistent and accurate information so health providers are able to inform those athletes who participate in high risk sports.

Female parents were more likely to agree that mouthguards should be mandatory for competitive youth soccer. Further analysis was done to assess other possible contributors to this finding. From our conceptual model, we felt that previous mouthguard use, previous injury, or health professional recommendations may influence the belief that mouthguards should be mandatory. However, we found no difference between male and female parents with respect to the proportion who stated that their doctor/physician recommended mouthguards, or who personally participated in youth sports. Conversely, male parents were more likely to report an orofacial injury and wear a mouthguard while participating in youth athletics than female parents. It is unclear if female parents are more cautious or more educated about orofacial injury in competitive youth soccer.

Barriers to mouth guard use reported in this study are similar to those previously reported: discomfort, peer pressure, and difficulty breathing. ${ }^{411}$ Most of these barriers can be addressed by the use of custom fit mouthguards. ${ }^{11} 22$ However, fitting athletes with custom mouthguards requires a visit to the dentist and can be relatively expensive (80-100 US\$). Although cost was not a major barrier in our study, it may be a barrier in a different population or if parents were asked about the cost of mouthguards over their child's playing career. Parents reported not having a mandatory mouthguard rule as a barrier to mouthguard use. The reason behind this finding is not clear. Parents may feel that peer pressure or athlete resistance to using mouthguard may be improved with compulsory use, however, further study is needed to truly understand this issue.

Most parents agreed mandatory mouthguard use reduces orofacial injury. However, only $32 \%$ of parents believed that orofacial injury is a significant problem and only $51 \%$ thought that mouthguards should be mandatory for competitive youth soccer. Put another way, parents seem to believe the risk of injury is not worth the "cost" of mouthguard use. To realize the true benefit of mouthguard use in competitive youth soccer, research efforts need to focus on understanding the incidence, econometrics, and morbidity of orofacial injuries in competitive youth soccer in addition to evaluating 


\section{Key points}

- Few competitive youth soccer athletes wear mouthguards.

- Little education is provided to parents regarding mouthguard use and soccer participation.

- Despite guidelines, few health professionals recommended mouthguards for their patients participating in competitive youth soccer.

the effectiveness of mouthguard use in competitive youth soccer.

Finally, very little educational material was provided to parents surveyed and most was verbal from the coach or other parents. Educational approaches should be developed and implemented by both youth soccer associations and health professionals. If more parents of competitive youth soccer know about orofacial injuries and their associated cost and morbidity, they may be more proactive in seeking advice from their physician or dentist.

\section{LIMITATIONS}

This study may be limited by the response rate of $48 \%$. The response rate may have been due to the rather novel approach for this survey, the world wide web. After pilot testing, most parents stated that an email, web based survey would provide them with the most convenient method to complete this study. Almost all parents had useable email addresses. However, we did not assess the various connection speeds that parents had to the web nor the capabilities of their personal computers. Complex computer language may have caused some computers to "crash" and discourage survey completion. This is a known limitation for web based surveys and care was made to limit the survey length and graphics. ${ }^{23}$ Also, we were unable to assess the number of parents who had an email filter that eliminates email from unknown sources. The response rate may also make the findings misleading as those who did not respond may be more apathetic to mouthguard use and the issues of orofacial injury in competitive youth soccer.

Other factors that may influence injury such as playing conditions, participant behavior during competitions, and refereeing variations were not assessed during our study. Our population of athletes had to "try-out" for their position on the roster and this factor may have confounded our results making them less generalizable to other less competitive leagues. The athletes in this study and their parents may have cared less about safety and more about competition and thus may have been more aggressive. Controlling aggressive behavior of youth athletes by improved refereeing may provide significant risk reduction in this age group as these issues may contribute to increasing the incidence of orofacial injury in competitive youth soccer. More importantly, these issues may be legitimate areas for possible intervention to reduce the burden of injury in this population. Further research in these areas is encouraged.

Finally, this study was performed during one season in a specific geographical region of the country and the results may not be generalizable to other populations.

\section{CONCLUSION}

Very few competitive youth soccer athletes use mouthguards. Health professional recommendations may influence parental beliefs regarding mandatory mouthguard use. However, very few health professionals recommend mouthguards for these youth athletes. Parents seem to understand the effectiveness of mouthguards in reducing orofacial injuries but only half believe that mouthguards should be mandatory for youth soccer. Educational efforts are needed to increase the awareness of parents to the potential high cost and morbidity of orofacial injuries in youth soccer so that parents can make informed decisions regarding mouthguard use for their competitive youth athlete. In addition, health professionals need to be consistent in their recommendations and realize opportunities for simple interventions during limited interaction with this population of patients.

\section{ACKNOWLEDGEMENT}

Dr Pribble was supported by the Robert Wood Johnson Foundation and the VA. This study was presented at the Pediatric Academic Society's Annual Meeting, May 2003, Seattle, Washington.

\section{Authors' affiliations}

J M Pribble, Department of Emergency Medicine Injury Research Center, Robert Wood Johnson Clinical Scholars Program and VA Medical Center, University of Michigan, Ann Arbor, Michigan

R F Maio, Department of Emergency Medicine Injury Research Center, University of Michigan, Ann Arbor, Michigan

G L Freed, Division of General Pediatrics, Child Health and Evaluation Research Unit, University of Michigan, Ann Arbor, Michigan

\section{REFERENCES}

1 Hoy K, Lindblad BE, Terkeslsen CJ. European soccer injuries: a prospective epidemiological and socioeconomic study. Am J Sports Med 1992;20:318-22

2 US Consumer Product Safety Commission. NEISS estimates query builder. Available at: http://63.74.109.4:7778/pls/portal30/PORTAL30. wwa_app_module.show?p sessionid $=10965$ (accessed 25 September 2003)

3 Ferrari CH, Ferreria de Mederios JM. Dental trauma and level of information: mouthguard use in different contact sports. Dent Traumatol 2002;18:144-7.

4 Yamada T, Sawaki Y, Tomida S, et al. Oral injury and mouthguard usage by athletes in Japan. Endod Dent Traumatol 1998;14:84-7.

5 Kvittem B, Hardie NA, Roettger M, et al. Incidence of orofacial injuries in high school sports. J Public Health Dent 1998;58:288-93.

6 Sane J, Ylipaavalniemi P. Maxillofacial and dental soccer injuries in Finland. Br J Oral Maxillofac Surg 1987;25:383-90.

7 Labella CR, Smith BW, Sigurdsson A. Effect of mouthguards on dental injuries and concussions in college basketball. Med Sci Sports Exerc 2002;34:41-4.

8 Maestrello-de Moya MG, Primosch RE. orofacial trauma and mouth-protector wear among high school varsity basketball players. Journal of Dentistry for Children 1989;56:36-9.

9 Flanders RA, Bhat $M$. The incidence of orofacial injuries in sports: a pilot study in Illinois. J Am Dent Assoc 1995; 126:491-6.

10 American Academy of Pediatric Dentistry. Mouth protectors. Available at: http://www.aapd.org/publications/brochures/mouthpro.asp (accessed 25 September 2003).

11 Ranalli DN. Prevention of sports-related traumatic dental injuries. Dent Clin North Am 2000;44:35-51, v-vi.

12 Nowjack-Raymer RE, Gift HC. Use of mouthguards and headgear in organized sports by school-aged children. Public Health Rep 1996;111:82-6.

13 Gardiner DM, Ranalli DN. Attitudinal factors influencing mouthguard utilization. Dent Clin North Am 2000;44:53-65.

14 Diab N, Mourino AP. Parental attitudes toward mouthguards. Pediatr Dent 1997; 19:455-60.

15 Maestrello CL, Mourino AP, Farrington FH. Dentists' attitudes towards mouthguard protection. Pediatr Dent 1999;21:340-6.

16 Kujala UM, Taimela S, Antti-Poika I, et al. Acute injuries in soccer, ice hockey, volleyball, basketball, judo, and karate: analysis of national registry data. BMJ 1995:311:1465-8.

17 Tuli T, Hachl O, Hohlrieder M, et al. Dentofacial trauma in sport accidents. Gen Dent 2002;50:274-9.

18 McBride CMEK, Lipkus IM. Understanding the potential of teachable moments: the case of smoking cessation. Health Education Research 2003;18:156-70.

19 Burt CW, Overpeck MD. Emergency visits for sports-related injuries. Ann Emerg Med 2001;37:301-8.

20 Mace SE, Gerardi MJ, Dietrich AM, et al. Injury prevention and control in children. Ann Emerg Med 2001;38:405-14.

21 Smith DM, Kovan JR, Rich BS, et al. Preparticipation physical evaluation. 2nd Ed. Minneapolis: The Physician and Sportsmedicine (McGraw-Hill), 1997

22 DeYoung AK, Robinson E, Godwin WC. Comparing comfort and wearability: custom-made vs self-adapted mouthguards. J Am Dent Assoc 1994; 125:1112-18.

23 Dillman DA. Internet and interactive voice response surveys. Mail and internet surveys. The tailored design method. 2nd Ed. New York: John Wiley, 2000:372-401. 